\title{
Behavior of a Jet Issuing Diagonally Upward into Two-Layer Density-Stratified Fluid in a Cylindrical Tank
}

\author{
Tomohiro Degawa ${ }^{*}$, Shota Fukue ${ }^{2}$, Tomomi Uchiyama ${ }^{1}$, Akira Ishikawa ${ }^{3}$, Koji Motoyama ${ }^{3}$ \\ ${ }^{1}$ Institute of Materials and Systems for Sustainability, Nagoya University, Nagoya, Japan \\ ${ }^{2}$ Mitsubishi Electric Co., Tokyo, Japan \\ ${ }^{3}$ Chubu Electric Power Co., Inc., Nagoya, Japan \\ Email: ^degawa@imass.nagoya-u.ac.jp
}

How to cite this paper: Degawa, T., Fukue, S., Uchiyama, T., Ishikawa, A. and Motoyama, K. (2017) Behavior of a Jet Issuing Diagonally Upward into Two-Layer Density-Stratified Fluid in a Cylindrical Tank. Journal of Flow Control, Measurement \& Visualization, 5, 51-64.

https://doi.org/10.4236/jfcmv.2017.53004

Received: January 6, 2017

Accepted: June 25, 2017

Published: June 28, 2017

Copyright () 2017 by authors and Scientific Research Publishing Inc. This work is licensed under the Creative Commons Attribution International License (CC BY 4.0).

http://creativecommons.org/licenses/by/4.0/

\begin{abstract}
This study is concerned with the experimental investigation of a jet issuing diagonally upward into a two-layer density-stratified fluid in a cylindrical tank and the resulting mixing phenomena. The upper and lower fluids are water and an aqueous solution of sodium chloride $(\mathrm{NaCl})$, respectively, and the lower fluid issues from a nozzle on the bottom of the tank. The angle between the centerline of the jet and the bottom of the tank is $60^{\circ}$, and the mass concentration of the $\mathrm{NaCl}$ solution is 0.02 . The investigation reveals that secondary flow is caused by the jet in the horizontal cross-sections of the tank and that it is composed of a pair of vortices. It confirms that the secondary flow at the density interface corresponds to an internal density current. The investigation also clarifies the effect of the Reynolds number of the jet on mixing between the lower and upper fluids.
\end{abstract}

\section{Keywords}

Jet, Density-Stratified Fluid, Mixing, Secondary Flow, Density Current

\section{Introduction}

As the density of liquefied natural gas (LNG) is specific to the production area and purification plant, density stratification is sometimes occurs when one batch of LNG is loaded on top of another batch of LNG in the same storage tank. The density of such stratified LNG changes owing to heat input from the outside of the tank. Thus, sudden mixing, known as rollover, occurs when the density of the lower LNG layer becomes lower than that of the upper layer. Rollover generates large amounts of vaporized gases, which cause severe damage to the storage 
tank. Therefore, the prevention and elimination of LNG stratification is essential for the operation of LNG storage tanks.

Mixing phenomena of density-stratified fluids in tanks have been studied via laboratory-based experiments. Mixing induced by a jet issuing from a nozzle mounted on the top or bottom of the tank has been investigated [1] [2] [3] [4]. Mixing LNG by a jet issuing from a nozzle on the bottom of the tank is considered a promising technique for preventing and eliminating stratification in LNG storage tanks. The current authors [5] performed a laboratory-based experiment on a two-layer density-stratified fluid in a rectangular tank. The upper and lower fluids were water and an aqueous solution of sodium chloride $(\mathrm{NaCl})$, respectively, and the lower fluid issued vertically upward from a nozzle on the bottom of the tank. The authors confirmed that the behavior of the jet is determined by the Reynolds number of the jet and the concentration of the lower fluid. The authors [6] also proposed a simulation method for a jet issuing into a two-layer density-stratified fluid. This method was based on a vortex in cell method, which discretizes the vorticity field into vortex elements and computes the temporal evolution of the flow by tracing the convection of each vortex element. The authors [6] performed the jet simulation under the same conditions as in their previous experiment [5] and confirmed the validity of the simulation method by comparing the simulated results with the experimental results.

The authors' abovementioned experiment and simulation were performed to investigate the behavior of a jet issuing vertically upward from a nozzle on the bottom of a rectangular tank. LNG storage tanks have a cylindrical shape, and a jet frequently issues diagonally upward from a nozzle on the bottom of the tank. In a cylindrical tank, a jet colliding with the wall of the tank is considered to flow along the wall, producing circulating flow in the tank. Although such induced flow differs completely from the flow in a rectangular tank, it has not been investigated.

In this study, laboratory experiments are conducted to investigate the behavior of a jet issuing into a two-layer density-stratified fluid in a cylindrical tank used to simulate an LNG storage tank. The upper and lower fluids are water and an aqueous solution of $\mathrm{NaCl}$, respectively, and the lower fluid issues diagonally upward from a nozzle on the bottom of the tank. The angle between the centerline of the nozzle and the bottom of the tank is set at $60^{\circ}$ with reference to LNG storage tanks. This study is performed at various jet velocities, because the velocity is only the variable for the operation of LNG storage tanks. The flows in the central vertical cross-section of the tank, as well as in horizontal cross-sections, are visualized to clarify the effects of the Reynolds number of the jeton the behavior of the jet and mixing phenomena.

\section{Experiment}

\subsection{Experimental Setup}

A schematic of the experimental setup using a cylindrical tank is shown in Figure 1 . The tank is made of transparent acrylic resin to enable visualization of the 


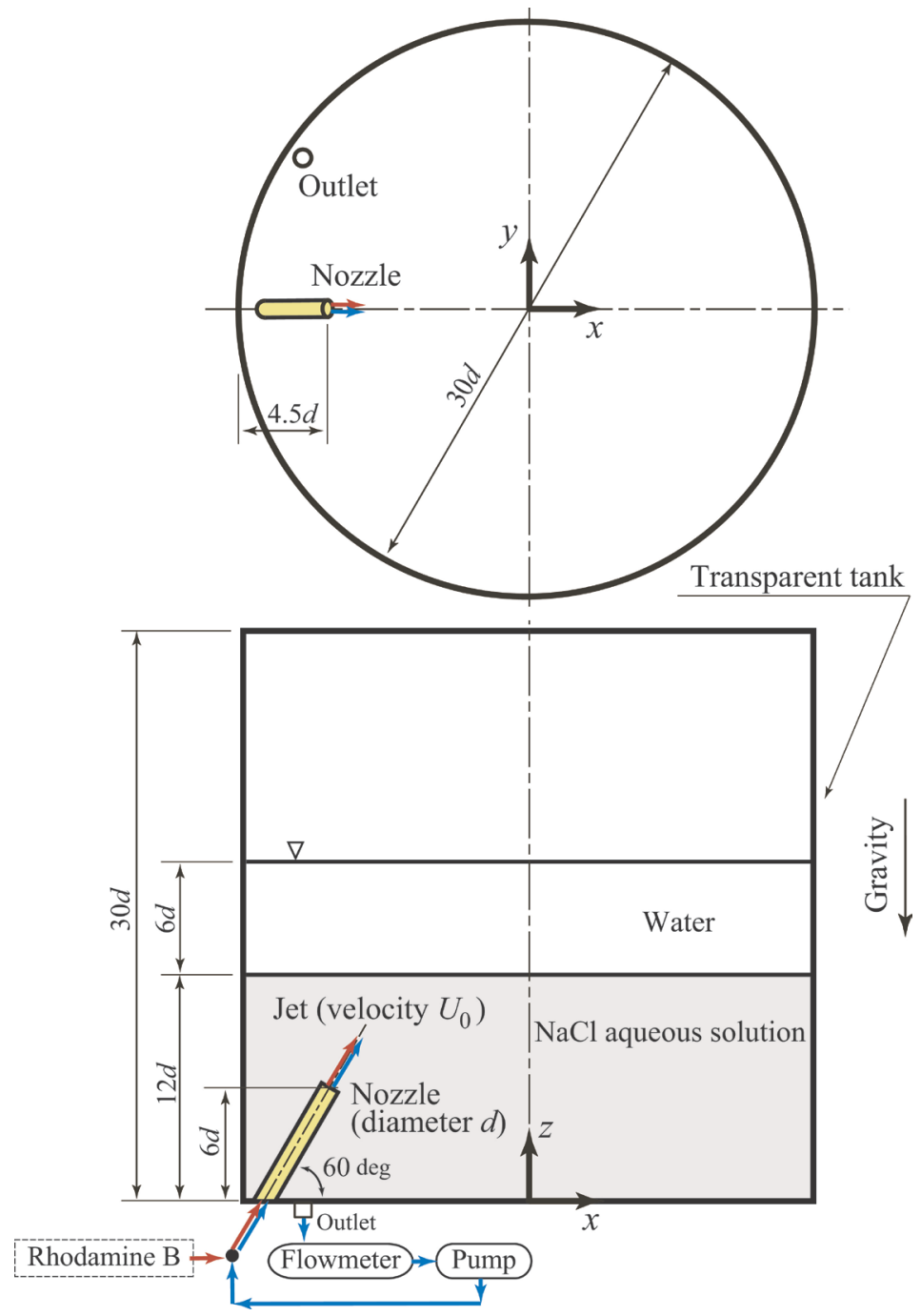

Figure 1. Schematic of experimental setup.

flow. The diameter and height are both $300 \mathrm{~mm}$. The origin of the coordinates is set at the center of the tank bottom with the $x$ - $y$ plane horizontal and the $z$-axis vertical. A nozzle is mounted on the tank bottom near the side wall of the tank. The inner diameter $d$ is $10 \mathrm{~mm}$, and the angle between the centerline of the nozzle and the $x$-axis is $60^{\circ}$. The nozzle outlet is positioned $4.5 d$ away from the tank wall and $6 d$ above the tank bottom. The nozzle is connected to a circular hole in the tank bottom via a tube, and a pump and a flowmeter are installed between the hole and the nozzle.

Initially (at $t=0$ ), a two-layer density-stratified fluid is in a static condition in the tank. The upper and lower fluids are water and an aqueous solution of $\mathrm{NaCl}$, respectively. The vertical thicknesses of the upper and lower fluids are $6 d$ and $12 d$, respectively. The mass concentration of the aqueous solution of $\mathrm{NaCl}$ is 0.02 .

\subsection{Experimental Method}

At a time $t>0$, the lower fluid (aqueous solution of $\mathrm{NaCl}$ ) issues from the noz- 
zle. The mean velocity in the nozzle outlet section is denoted by $U_{0}$. The volume of the fluid in the tank is maintained at a constant level using pump-driven fluid circulation.

To visualize the flows in the central vertical and horizontal cross-sections, a small amount of fluorescent dye (Rhodamine B) is added to the jet. Images of the vertical and horizontal cross-sections are captured by a video camera using a laser light sheet (power: $1 \mathrm{~W}$, wavelength: $532 \mathrm{~nm}$, thickness: $2 \mathrm{~mm}$ ). The spatial resolution, framerate, and shutter speed of the camera are $640 \times 480$ pixels, 200 fps, and $1 / 200$ s, respectively.

The Reynolds number of the jet Re is defined by $d U_{0} / v$, where $v$ is the kinematic viscosity of water. The experiments are conducted at values of $475 \leq \mathrm{Re} \leq$ 4753.

The experimental conditions are listed in Table 1.

\section{Results and Discussions}

\subsection{Jet Behavior Relative to Density-Interface and Water Surface}

Figure 2 shows a visual image of the central vertical cross-section of the tank. The white area, which represents the fluorescent dye issuing from the nozzle, reveals the location of the jet. Using such images, the temporal variation of the

Table 1. Experimental conditions.

\begin{tabular}{cc} 
Upper fluid & Water \\
\hline Lower fluid & Aqueous $\mathrm{NaCl}$ \\
Nozzle diameter & $10 \mathrm{~mm}$ \\
Angle of nozzle to horizontal $(x)$ axis & $60^{\circ}$ \\
Tank diameter & $30 d$ \\
Reynolds number $\mathrm{Re}=d U_{0} / v$ & $475 \leq \mathrm{Re} \leq 4753$ \\
Concentration of aqueous $\mathrm{NaCl}$ & 0.02 \\
Thickness of upper fluid & $6 d$ \\
Thickness of lower fluid & $12 d$ \\
\hline
\end{tabular}

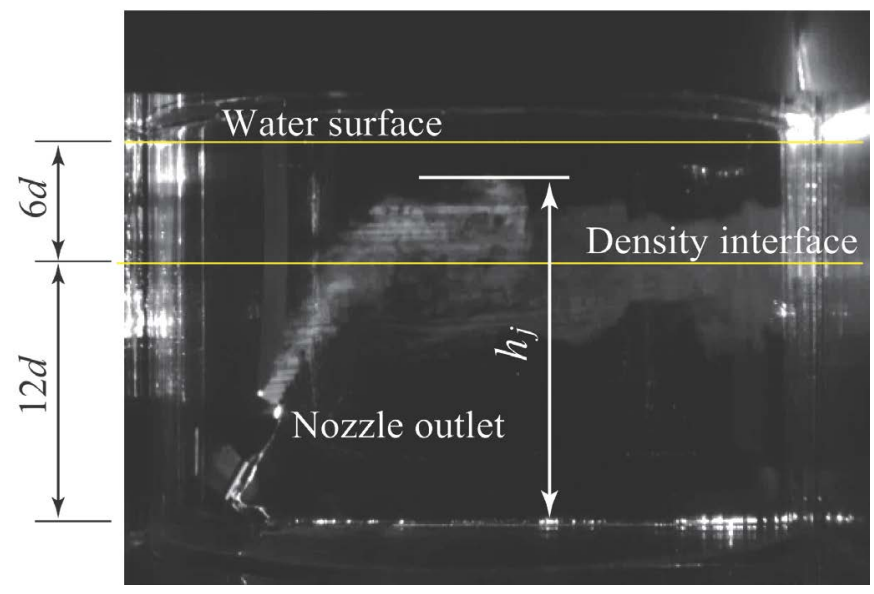

Figure 2. Visual image showing the definition of the jet height $h_{j}$ 
jet height $h_{j}$ is measured. This study defines the nondimensional height as $H_{j}=$ $\left(h_{j}-12 d\right) / d$, where $h_{j}-12 d$ is the height from the density interface. Figure 3 shows the relationship between $H_{j}$ and Re, where the relationship at a nondimensional time $\dot{t}\left(=t U_{0} / d\right)$ of 500 and 2000 are compared. For every Re value, $H_{j}$ is positive, which indicates that the jet reaches the density interface. $H_{j}$ increases with an increase in Re. When Re is larger than a critical value $\mathrm{Re}_{\mathrm{cr}}$, the jet reaches the water surface $\left(H_{j}=6\right)$. The $\operatorname{Re}_{\text {cr }}$ values at $t^{*}=500$ and 2000 are 3089 and 2614 , respectively.

When the jet height $h_{j}$ is less than $13 d$ or $H_{j} \leq 1$, the jet is regarded as having reached the density interface without penetrating it. In this case, the jet behavior is classified into three patterns, namely, A, B, and C, according to the Re value.

- Pattern A: The jet reaches the interface without penetrating it but spreads almost horizontally outward along the interface.

- Pattern B: The jet penetrates the interface but does not reach the upper water surface. The top of the jet falls back to the interface and spreads horizontally without penetrating the interface again.

- Pattern C: The jet reaches the upper water surface, spreads along the surface, and falls back to the interface.

Table 2 lists the patterns of jet behavior (Patterns A, B, and C) for different Re values at $t=2000$.

Figure 4 shows images of the jet at $t^{*}=500$ visualized in the central vertical cross-section of the tank. Images for $\mathrm{Re}=475,2139$, and 3565 are compared. In the case when $\operatorname{Re}=475$, the jet behavior is classified as Pattern $\mathrm{A}$, where $H_{j}=$ 0.23 . The jet reaches the density interface $(z / d=12)$ and spreads almost horizontally along the interface. In a lower cross-section $(z / d=9)$, the top of the jet

Table 2. Classification of jet behavior.

\begin{tabular}{cccccccccc}
\hline Re & 475 & 713 & 951 & 1188 & 1426 & 1663 & 1901 & 2139 & 2376 \\
Pattern & A & A & B & B & B & B & B & B & B \\
Re & 2614 & 2852 & 3089 & 3565 & 4040 & 4753 & & & \\
Pattern & C & C & C & C & C & C & & & \\
\hline
\end{tabular}

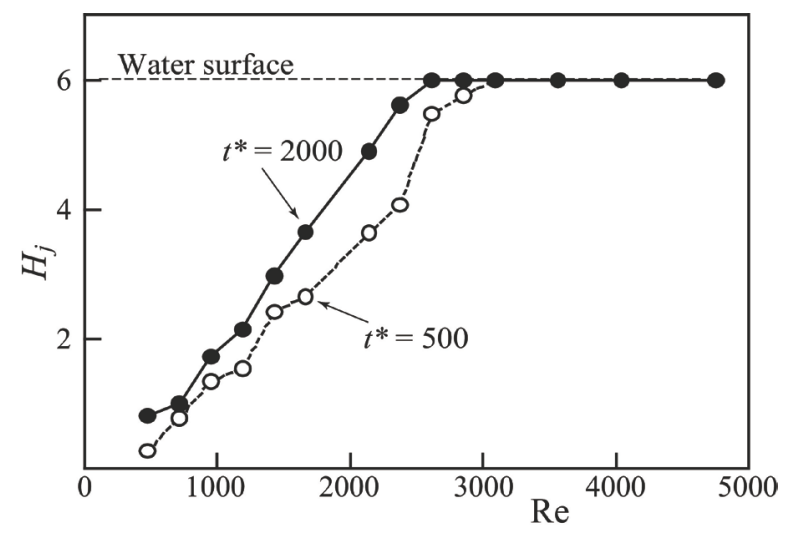

Figure 3. Relationship between Reynolds number Re and nondimensional jet height $H_{j}$ 

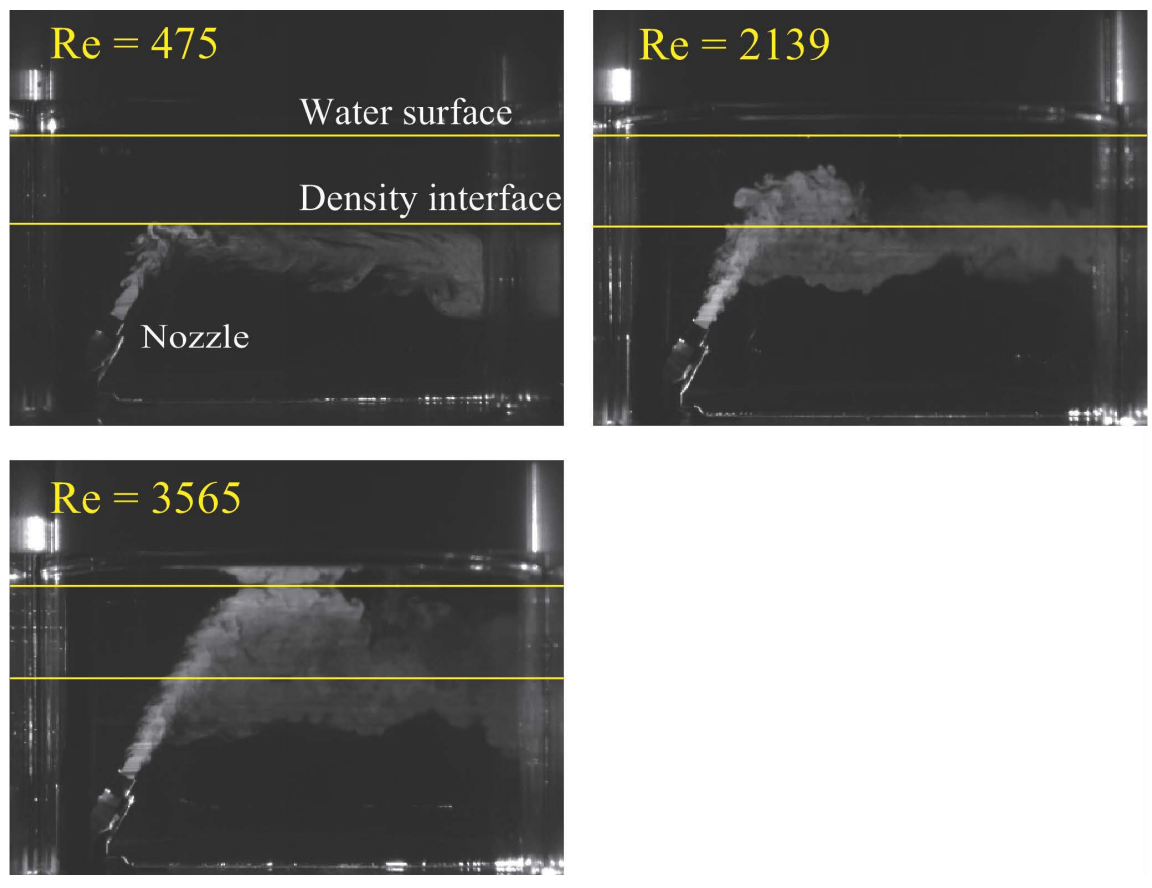

Figure 4. Flow visualized in central vertical cross-section of tank at $t^{*}=500$.

collides with the tank wall, flows along the tank wall, and returns to the position where the jet penetrates the cross-section, producing a pair of vortices in the horizontal cross-section, as is explained later. In the case when $\mathrm{Re}=2139$, the jet behavior is classified as Pattern B, where $H_{j}=3.63$. The top of the jet penetrates the density interface and falls back to the interface owing to the gravitational effect, as the density of the jet is greater than the density of the upper fluid. The fluid descending from the top of the jet spreads horizontally when it again reaches the density interface. This downward flow causes the interface to heave and leads to mixing of the fluids in the layer region along the interface. The jet reaching the density interface flows almost horizontally along the tank wall, as is explained later. In the case when $\mathrm{Re}=3565$, the jet behavior is classified as Pattern $\mathrm{C}$, where $H_{j}=6$. The jet spreads horizontally just below the water surface. Mixing of fluids around the density interface is also observed. The flow colliding with the tank wall spreads markedly in a vertical direction along the wall. Active mixing is generated in wider regions.

The time $t_{1}$ when the top of the jet reaches the water surface is measured using visualized images of the flow in the central vertical cross-section of the tank. Figure 5 shows the relationship between the nondimensional time $t_{1}^{*}=t_{1} U_{0} / d$ and Re. The value of $t_{1}^{*}$ decreases with an increase in Re. A marked decline occurs at $\operatorname{Re} \geq 3089$. This is because the top of the jet falls back in the upper layer when $\operatorname{Re} \leq 2852$, but it reaches the water surface without falling when $\operatorname{Re} \geq 3089$. Such behavior of the top of the jet is confirmed by the images for $R e=2139$ and 3565, as shown in Figure 4.

\subsection{Spread of Jet}

Figure 6 shows visualized images of the flow for $\operatorname{Re}=475$ at the three time 


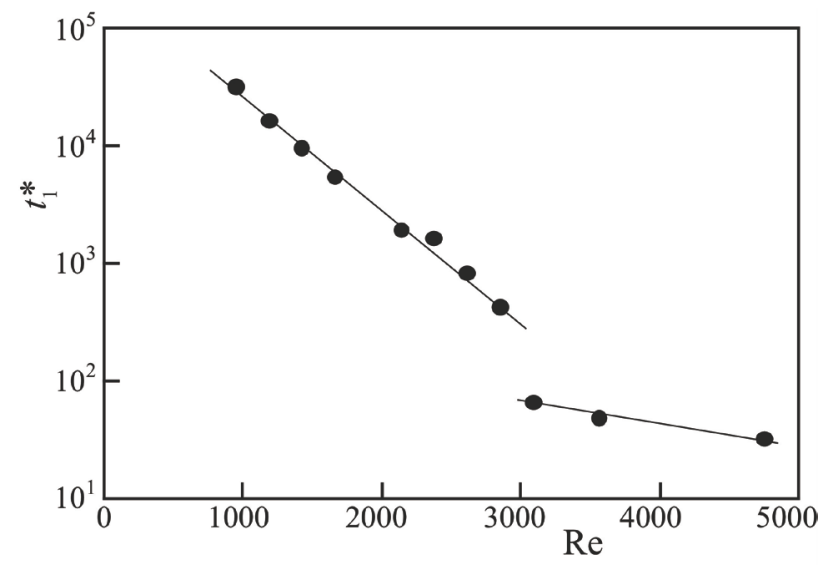

Figure 5. Effect of Re on time of arrival of jet at water surface $t_{1}^{*}$.

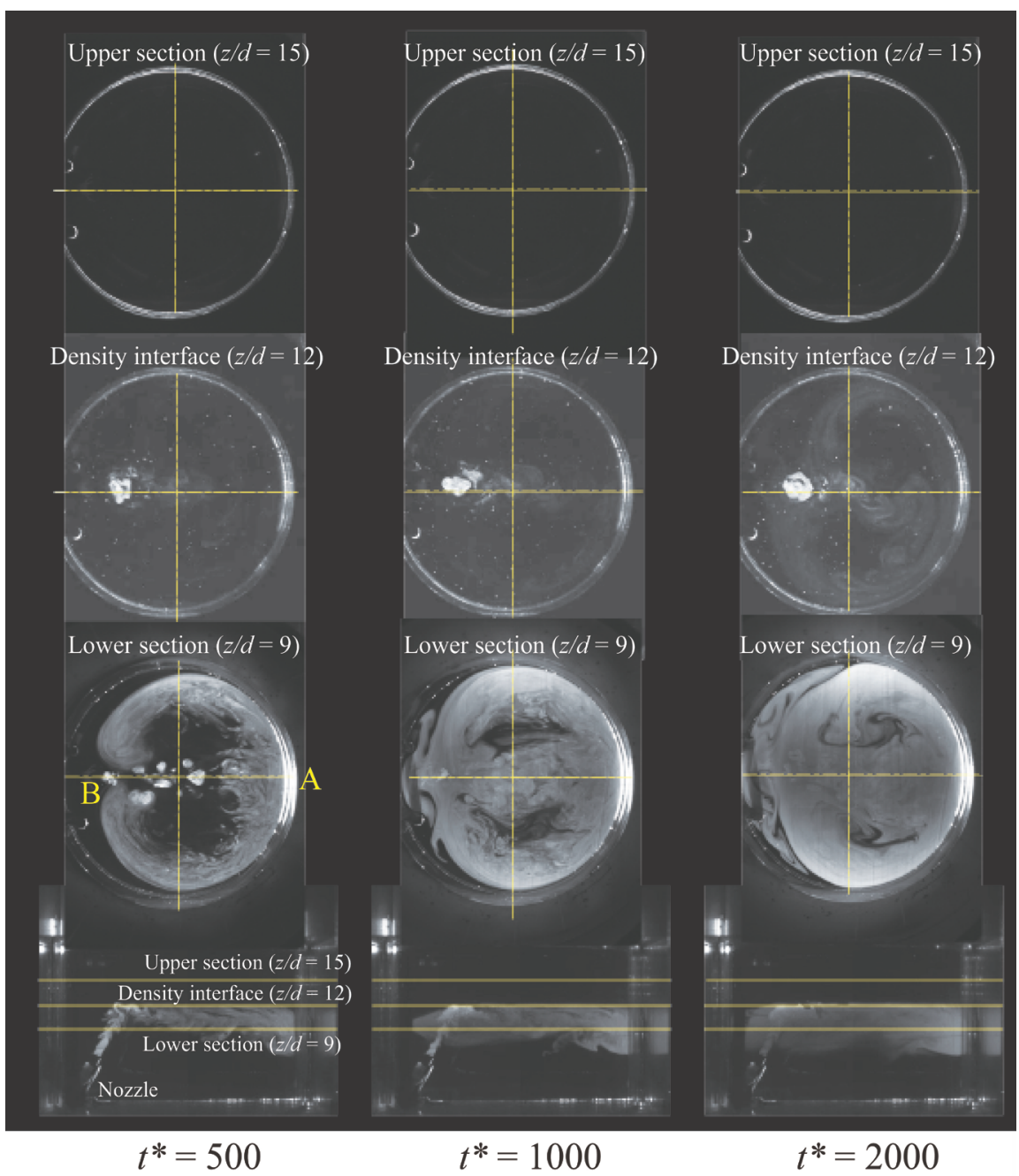

Figure 6. Flow visualized in central vertical and horizontal cross-sections at $\operatorname{Re}=475$.

points of $t^{*}=500,1000$, and 2000. Images in the central vertical cross-section $(x-Z$ section), in the horizontal cross-section at the density interface $(z / d=12)$, as well as in the lower and upper horizontal cross-sections $(z / d=9$ and 15) are 
shown. When $t^{*}=500$ in the lower cross-section at $z / d=9$, the jet colliding with the tank wall flows along the wall and returns to the position where the jet penetrates the cross-section. The locations of collision and penetration are indicated by $\mathrm{A}$ and $\mathrm{B}$, respectively. In this cross-section, a pair of vortices is produced. At $t^{*}=500$, the top of the jet appears in the cross-section at the density interface $(z / d=12)$, but it does not appear in the upper cross-section at $z / d=15$. When $t=1000$, the jet spreads widely with the production of a pair of vortices in the lower cross-section $(z / d=9)$. When $t=2000$, the fluid issuing from the nozzle occupies almost all of the lower cross-section $(z / d=9)$. In the cross-section at the density interface $(z / d=12)$, the collision of the jet with the tank wall and the spread of the jet along the tank wall are also confirmed. However, the spread of the jet is less than that in the lower fluid. The jet does not reach the upper cross-section at $z / d=15$. These findings demonstrate that mixing is mainly produced by the jet in the lower cross-section.

Figure 7 shows visualized images for $\mathrm{Re}=2139$. In the lower cross-section at $z / d=9$ and the density interface at $z / d=12$, one can observe the collision of the jet with the tank wall and the spread of the jet along the wall. The spreads of the

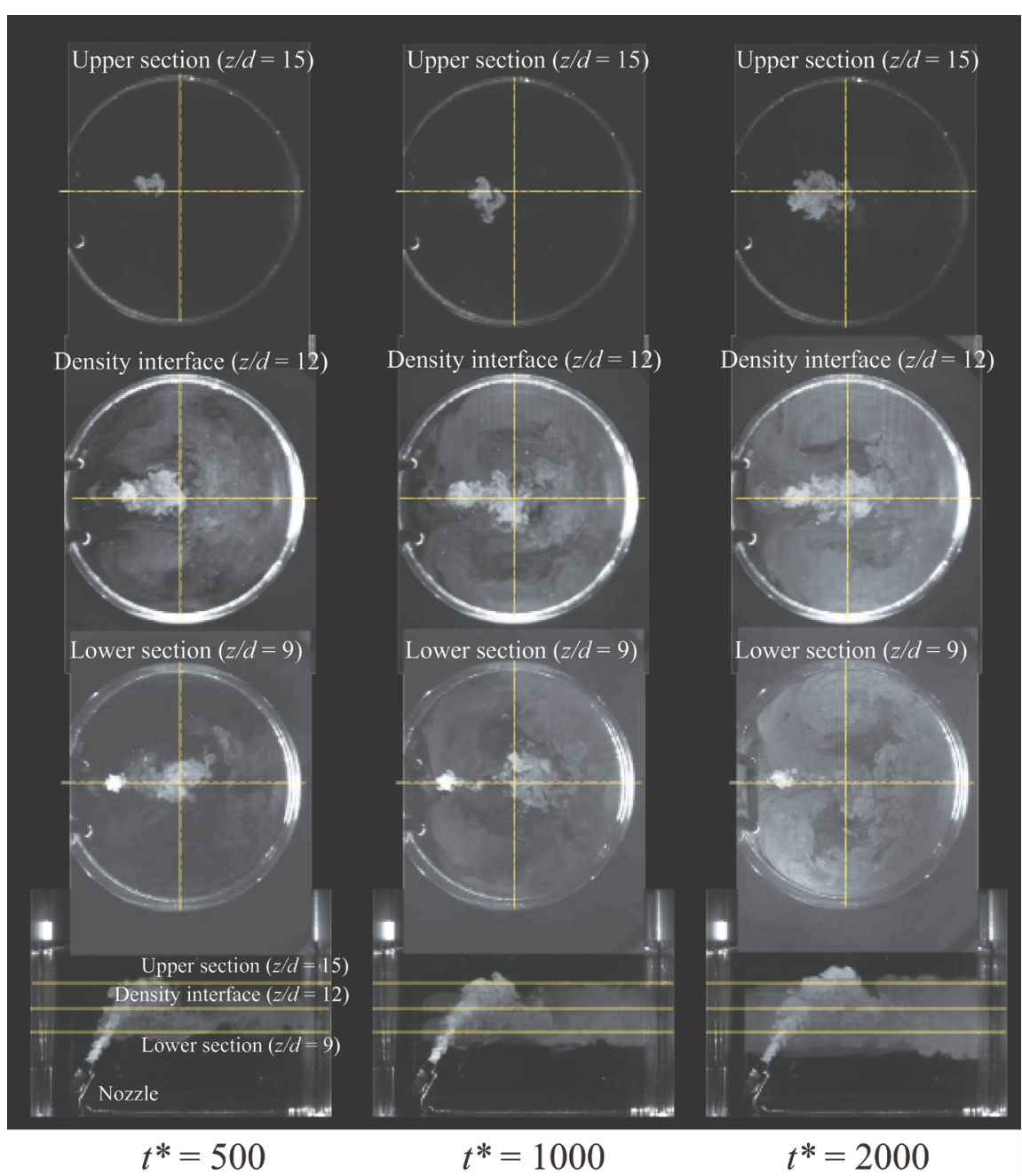

Figure 7. Flow visualized in central vertical and horizontal cross-sections at $\operatorname{Re}=2139$. 
jet in these cross-sections are almost the same. However, the spread at the density interface is greater when compared with the result for $\mathrm{Re}=475$ shown in Figure 6. In the upper cross-section at $z / d=15$, only the top of the jet reaches this point, and therefore spreading of the jet hardly occurs. It is found that the jet gives rise to mixing of fluids below the density interface.

Visualized images for $\mathrm{Re}=3656$ are shown in Figure 8. Active spread of the jet within the density interface $(z / d=12)$ occurs at every time point. In the lower and upper cross-sections $(z / d=9$ and 15$)$, the jet spreads with the lapse of time. It is possible to observe the progress of mixing produced by the jet in the entire region of the tank. It should be noted that mixing scarcely occurs in the lower fluid layer according to the flow visualizations shown in Figures 6-8, and therefore it can be considered that only the lower fluid is always issued from the nozzle. Therefore, the mass flowrate remains unaltered at every Re.

When a fluid with a density of $\rho$ reaches a density interface from the outside, it flows in a horizontal direction along the interface if the condition $\rho_{1}<\rho<\rho_{2}$ is satisfied, where $\rho_{1}$ and $\rho_{2}$ are the densities of the upper and lower fluids, respectively [7]. Such flow is recognized as the intrusion of an internal density current.

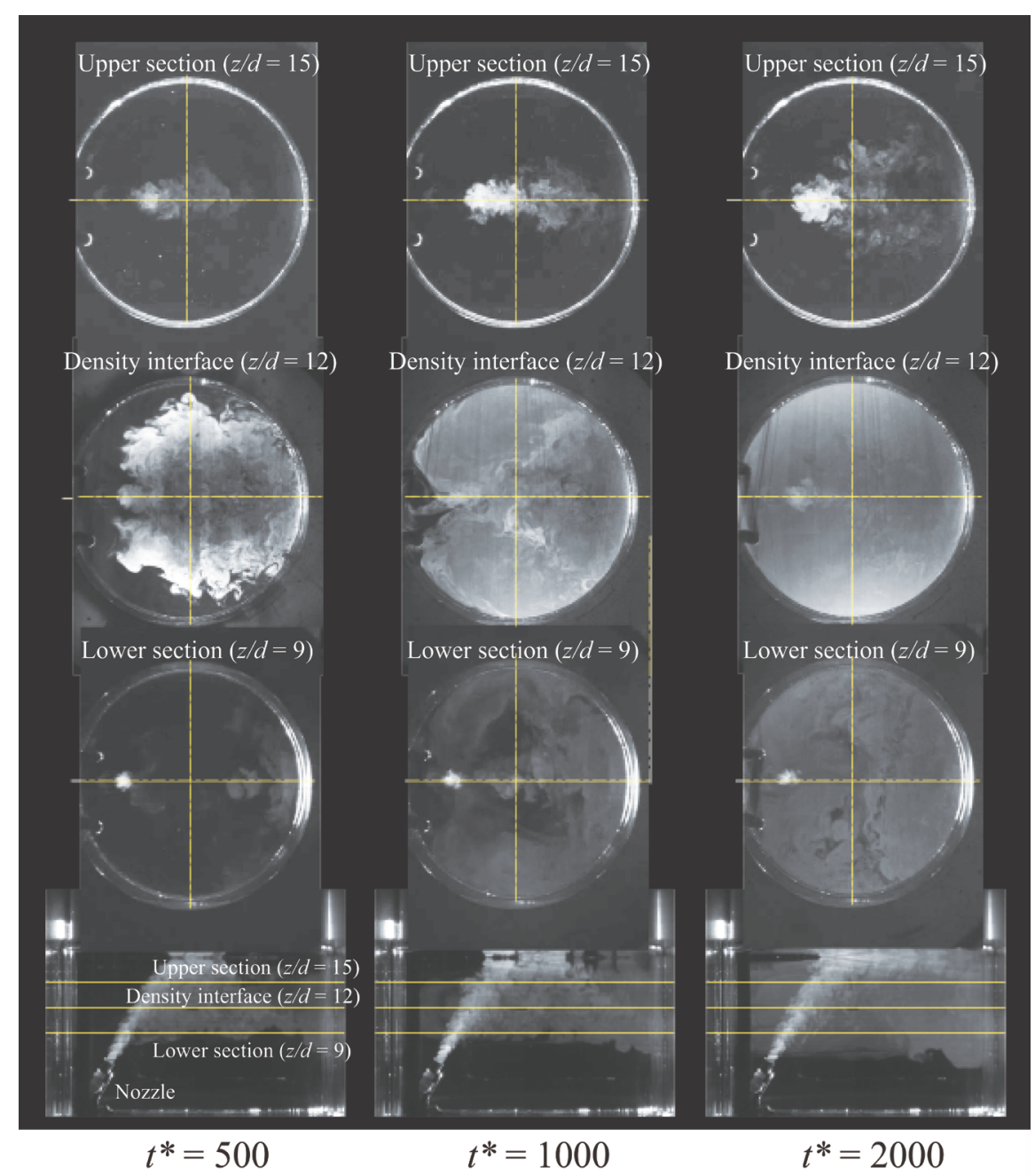

Figure 8. Flow visualized in central vertical and horizontal cross-sections at $\operatorname{Re}=3565$. 
An example of this type of flow can be observed in the temperature-stratified water of a dam reservoir when muddy water mixed with sand flows into the reservoir and reaches the density interface. The horizontal flow along the density interface visualized in Figures 6-8 corresponds to the intrusion of an internal density current. Such flow was also revealed by the authors' experiment [5] and simulation [6] for a jet issuing vertically upward into a two-layer density-stratified fluid in a rectangular tank. The present study confirms that the internal density current induced by a jet in a cylindrical tank collides with the tank wall and forms a pair of vortices in the horizontal cross-section.

\subsection{Temporal Variation of Distribution of Fluorescent Dye Issuing with the Jet}

The visualized images of the flow in each horizontal cross-section at $t^{*}=500$ and 2000 shown in Figures 6-8 are divided into grids of $0.608 d \times 0.608 d$ to determine the luminance of the fluorescent dye $\Gamma$ at each grid point by 256 gradations. The distribution of $\Gamma$ along the horizontal axes ( $x$ - and $y$-axes) is measured. Figure 9 shows the distribution in the lower cross-section $(z / d=9)$ for $\operatorname{Re}=$ 475. $\Gamma$ along the $y$-axis has a maximum value near the tank wall at $y / d= \pm 15$. This is attributable to the fact that the jet flows along the tank wall, as demonstrated in Figure 6. When $t=2000$, the maximum value increases and the $\Gamma$ value becomes higher around the tank center $(y=0)$. The progress of mixing can be reconfirmed. The $\Gamma$ value along the $x$-axis reaches the maximum at several points when $t=500$. This is because the centerline of the jet coincides with the $x$-axis, and therefore the top of the jet heaves with the convection. However, the distribution of $\Gamma$ becomes flatter when $t^{*}=2000$, because the fluid issuing from the nozzle is distributed almost uniformly in the cross-section.

Figure 10 shows the distribution of $\Gamma$ in the cross-section of the density interface $(z / d=12)$ for $\operatorname{Re}=2139$. The $\Gamma$ value along the $y$-axis is higher around the centerline of the tank $(x=0)$. This is because the jet penetrates the density interface, as shown in Figure 7. The $\Gamma$ value along the $x$-axis reaches the maximum at $y / d \simeq-6$. This is due to the penetration of the jet.

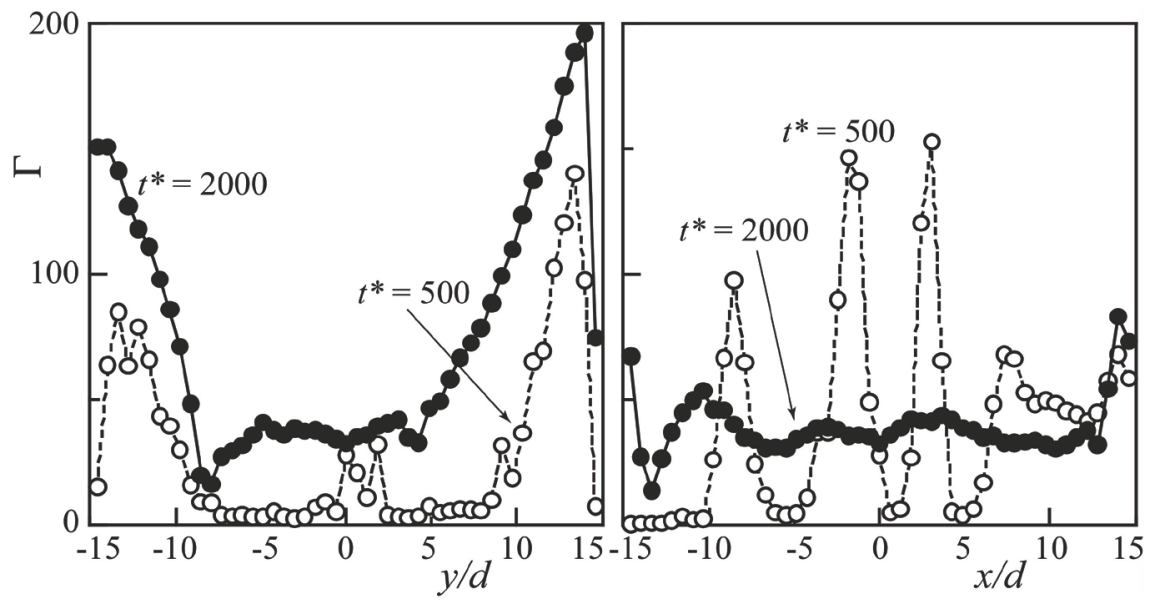

Figure 9. Distribution of $\Gamma$ in lower cross-section $(z / d=9)$ at $\operatorname{Re}=475$. 


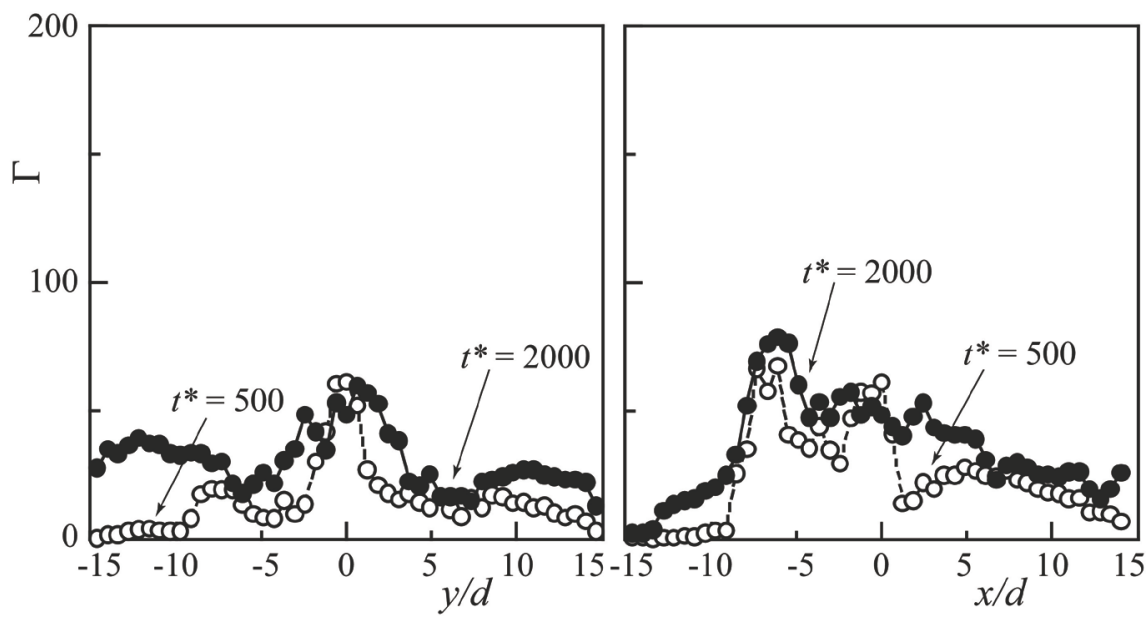

Figure 10. Distribution of $\Gamma$ at density interface $(z / d=12)$ at $\operatorname{Re}=2139$.

The distribution of $\Gamma$ at the density interface $(z / d=12)$ for $\mathrm{Re}=3565$ is shown in Figure 11. The $\Gamma$ value along the $y$-axis reaches the maximum value near the tank wall $(y / d= \pm 15)$ when $t=500$. This is because the jet penetrating the density interface flows along the tank wall, and accordingly a main stream (a highvelocity region) is present near the wall. When $t=2000, \Gamma$ increases on approaching the wall, but the distribution is flatter than that when $t=500$. The distribution of $\Gamma$ along the $x$-axis is almost the same as that when $\operatorname{Re}=2139$, but the $\Gamma$ value is lower. This is because the top of the jet is in the upper fluid layer.

The spatial average value of $\Gamma$, namely $\langle\Gamma\rangle$, is calculated for the three horizontal cross-sections at $z / d=9,12$, and $15 .\langle\Gamma\rangle$ varies with the lapse of time, as shown in Figure 12, where the cross-section at $z / d=12$ corresponds to the density interface. For $\operatorname{Re}=475,\langle\Gamma\rangle$ is higher in the lower cross-section at $z / d=$ 9,but it is extremely low in the other cross-sections. This indicates that the jet spreads only in the lower fluid. For $\operatorname{Re}=2138,\langle\Gamma\rangle$ becomes larger with the lapse of time. The rate of change with time at the density interface $(z / d=12)$ is the highest, which demonstrates the active spread of the jet at the density interface. For $\mathrm{Re}=3565$, the jet spreads markedly at the density interface. However, $\langle\Gamma\rangle$ remains almost unchanged at $t^{*} \geq 300$, which indicates saturation of the spread of the jet. In the other cross-sections at $z / d=9$ and $15,\langle\Gamma\rangle$ increases with the lapse of time and mixing progresses.

\section{Conclusions}

A jet issues diagonally upward from a nozzle into a two-layer density-stratified fluid in a cylindrical tank. The upper and lower fluids are water and an aqueous $\mathrm{NaCl}$ solution, respectively, and the lower fluid issues from a nozzle mounted on the tank bottom. The nozzle diameter $d$ is $10 \mathrm{~mm}$, and the angle between the centerline of the jet and the bottom of the tank is $60^{\circ}$. The diameter of the tank is $30 d$. The thicknesses of the upper and lower fluids are $6 d$ and $12 d$, respectively. The mass concentration of the aqueous $\mathrm{NaCl}$ solution is 0.02 . The Reynolds number Re, defined by the nozzle diameter, the fluid velocity at the nozzle outlet, and the kinematic viscosity of water, range from 475 to 4753 . The results are 


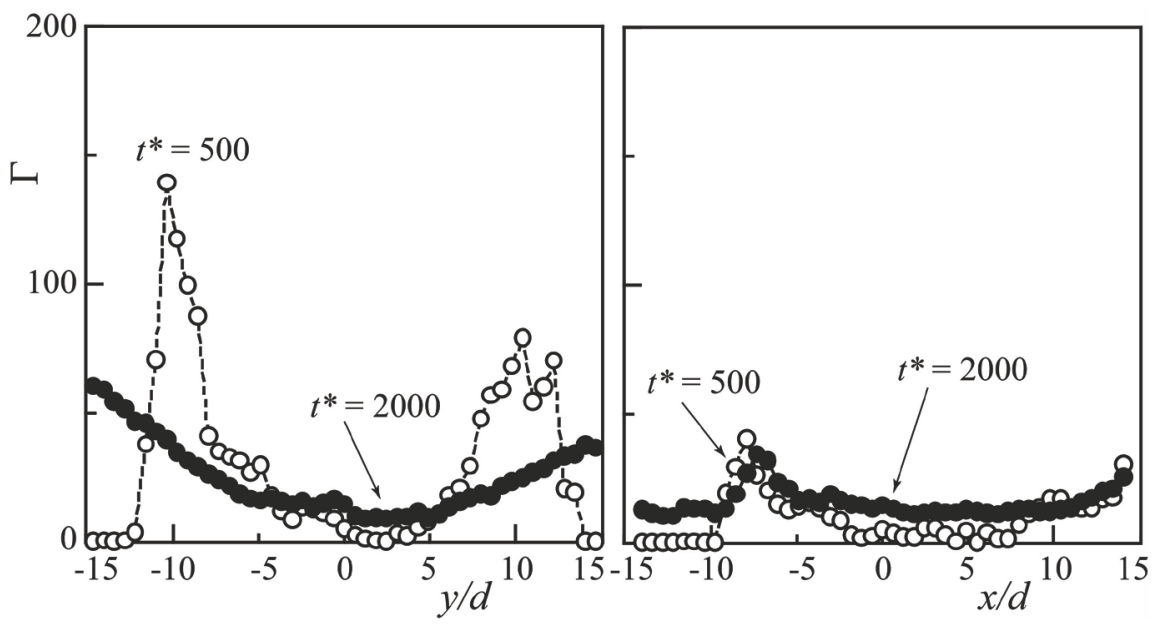

Figure 11. Distribution of $\Gamma$ at density interface $(z / d=12)$ at $\operatorname{Re}=3565$.
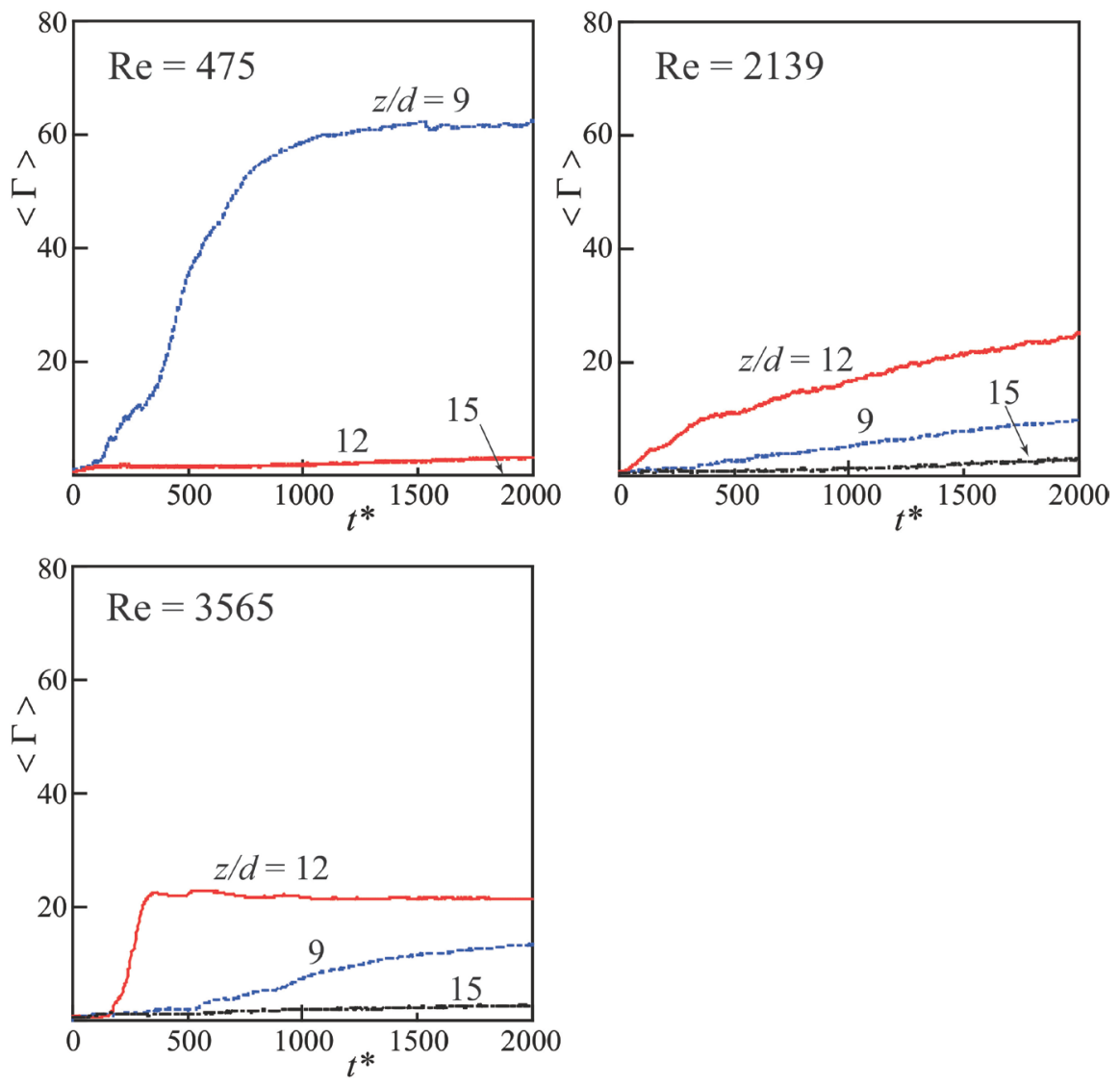

Figure 12. Temporal variation of spatially averaged values of $\Gamma$ at density interface and in horizontal cross-sections.

summarized as follows:

1) The jet causing the flows along the density interface collides with the tank wall and induces a pair of vortices at the interface. Jet-induced flow is also observed in a horizontal cross-section in the lower fluid. Such secondary flow noticeably appears when Re is higher or the jet markedly penetrates the density interface. 
2) The abovementioned flow along the density interface corresponds to an internal density current. This study reveals that an internal density current with a pair of vortices is induced by a jet issuing diagonally upward from a nozzle into a two-layer density-stratified fluid in a cylindrical tank.

3) With an increase in $R e$, the jet spreads more in the vertical direction and therefore mixing of fluids in the tank becomes more active.

\section{References}

[1] Baines, W.D., Turner, J.S. and Campbell, I.H. (1990) Turbulent Fountains in an Open Chamber. Journal of Fluid Mechanics, 212, 557-592.

https://doi.org/10.1017/S0022112090002099

[2] Bloomfield, L.J. and Kerr, R.C. (1998) Turbulent Fountains in a Stratified Fluid. Journal of Fluid Mechanics, 358, 335-356. https://doi.org/10.1017/S0022112097008252

[3] Lin, Y.J.P. and Linden, P.F. (2005) The Entrainment Due to a Turbulent Fountain at a Density Interface. Journal of Fluid Mechanics, 542, 25-52. https://doi.org/10.1017/S002211200500635X

[4] Ansong, J.K., Kyba, P.J. and Sutherland, B.R. (2008) Fountains Impinging upon a Density Interface. Journal of Fluid Mechanics, 595, 115-139. https://doi.org/10.1017/S0022112007009093

[5] Shakouchi, S., Fukue, S. and Uchiyama, T. (2015) Investigation of the Behavior of a Jet Issued into Two-Layer Density-Stratified Fluid, Journal of Flow Control, Measurement and Visualization, 3, 1-9. https://doi.org/10.4236/jfcmv.2015.31001

[6] Shakouchi, S., Shimada, S. and Uchiyama, T. (2015) Numerical Simulation of Jet Flow Issued into Density-Stratified Fluid by Vortex in Cell Method, Advances and Applications in Fluid Mechanics, 17, 115-134. https://doi.org/10.17654/15-AAFM-01701-115

[7] Simpson, J.E. (1982) Gravity Currents in the Laboratory, Atmosphere, and Ocean. Annual Review of Fluid Mechanics, 14, 213-234. https://doi.org/10.1146/annurev.fl.14.010182.001241 


\section{Nomenclature}

d nozzle diameter

$H_{j} \quad$ nondimensional height from density interface $=\left(h_{j}-12 d\right) / d$

$h_{j} \quad$ height of jet

Re Reynolds number $=d U_{0} / v$

$\operatorname{Re}_{\text {cr }} \quad$ Reynolds number at which the jet reaches water surface

$t \quad$ elapsed time from start of issuing jet

$t^{*} \quad$ nondimensional time $=t U_{0} / d$

$U_{0} \quad$ mean velocity at nozzle outlet

$x, y, z \quad$ orthogonal coordinates

$\Gamma \quad$ luminance of the fluorescent dye concentration

$\langle\Gamma\rangle \quad$ spatial averaged value of $\Gamma$

$v \quad$ kinematic viscosity of water

Submit or recommend next manuscript to SCIRP and we will provide best service for you:

Accepting pre-submission inquiries through Email, Facebook, LinkedIn, Twitter, etc. A wide selection of journals (inclusive of 9 subjects, more than 200 journals)

Providing 24-hour high-quality service

User-friendly online submission system

Fair and swift peer-review system

Efficient typesetting and proofreading procedure

Display of the result of downloads and visits, as well as the number of cited articles

Maximum dissemination of your research work

Submit your manuscript at: http://papersubmission.scirp.org/

Or contact jfcmv@scirp.org 